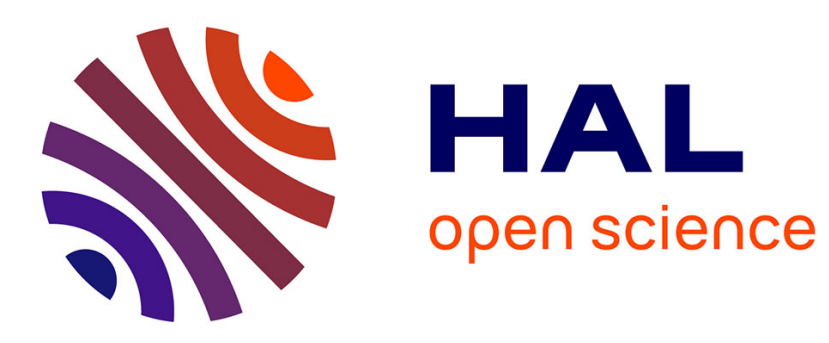

\title{
Why Microtubules Run in Circles: Mechanical Hysteresis of the Tubulin Lattice
}

\author{
Falko Ziebert, Herve Mohrbach, Igor M. Kulic
}

\section{To cite this version:}

Falko Ziebert, Herve Mohrbach, Igor M. Kulic. Why Microtubules Run in Circles: Mechanical Hysteresis of the Tubulin Lattice. Physical Review Letters, 2015, 114 (14), pp.148101. 10.1103/PhysRevLett.114.148101 . hal-01513174

\section{HAL Id: hal-01513174 \\ https://hal.univ-lorraine.fr/hal-01513174}

Submitted on 25 Apr 2017

HAL is a multi-disciplinary open access archive for the deposit and dissemination of scientific research documents, whether they are published or not. The documents may come from teaching and research institutions in France or abroad, or from public or private research centers.
L'archive ouverte pluridisciplinaire HAL, est destinée au dépôt et à la diffusion de documents scientifiques de niveau recherche, publiés ou non, émanant des établissements d'enseignement et de recherche français ou étrangers, des laboratoires publics ou privés. 


\title{
Why Microtubules Run in Circles: Mechanical Hysteresis of the Tubulin Lattice
}

\author{
Falko Ziebert, ${ }^{1,2}$ Hervé Mohrbach, ${ }^{3,2}$ and Igor M. Kulić ${ }^{2}$ \\ ${ }^{1}$ Albert-Ludwigs-Universität, 79104 Freiburg, Germany \\ ${ }^{2}$ Institut Charles Sadron UPR22-CNRS, 67034 Strasbourg, France \\ ${ }^{3}$ Groupe BioPhysStat, LCP-A2MC, Université de Lorraine, 57078 Metz, France
}

(Received 18 May 2014; published 6 April 2015)

\begin{abstract}
The fate of every eukaryotic cell subtly relies on the exceptional mechanical properties of microtubules. Despite significant efforts, understanding their unusual mechanics remains elusive. One persistent, unresolved mystery is the formation of long-lived arcs and rings, e.g., in kinesin-driven gliding assays. To elucidate their physical origin we develop a model of the inner workings of the microtubule's lattice, based on recent experimental evidence for a conformational switch of the tubulin dimer. We show that the microtubule lattice itself coexists in discrete polymorphic states. Metastable curved states can be induced via a mechanical hysteresis involving torques and forces typical of few molecular motors acting in unison, in agreement with the observations.
\end{abstract}

DOI: 10.1103/PhysRevLett.114.148101

PACS numbers: 87.16.Ka, 82.35.Pq, 87.15.-v

Microtubules (MTs) are the stiffest cytoskeletal component and play many versatile and indispensable roles in living cells. As "cellular bones," they define to a great extent cell mechanics, and are crucial for cellular transport and cell division [1-4]. Beyond their biological importance, MTs have been used as molecular sensors for intracellular forces, as biotemplates for nanopatterning, and as building blocks for hybrid materials and active systems like artificial cilia and self-propelled droplets [5-10]. The MT's structure is well known [11]: the elementary building blocks, tubulin dimers, polymerize head to tail into linear protofilaments that associate side by side to form the hollow tube structure known as the MT.

Despite the MTs' importance, widespread use, the knowledge of their structure, and numerous experiments probing their elastic properties [12-16], understanding their mechanics still poses challenging problems. A remarkable one is found in MT gliding assays [17-20], see Fig. 1: already two decades ago, Amos and Amos [18] observed that MTs driven by kinesin motors on a glass surface can form arcs which continue gliding for significant times before suddenly straightening out. They remarked with quite some foresight that these circular MT states could be explained by the existence of alternative tubulin dimer conformations [21]. The observation remained without wider public notice despite the frequent reoccurrence of MT arcs in the gliding dynamics of single filaments [22-24], bundles [25-27], and in collective (high density) gliding [28]. Force-induced circular arcs on the same scale, differing from classical buckling, have been found in other situations [19,29], also in vivo [30-32]. While the lifetime of MT rings varies, their characteristic size of about one micron is preserved in single filament experiments [18,22-24], indicating a robust mechanism at work. Internal degrees of freedom of the MT lattice (e.g., interprotofilament shear $[33,34]$ and tubulin conformational switching [35]) have been recently used to explain the surprising length dependent stiffness in clamped MT experiments $[15,16]$. However, long lived, highly curved states or rings could not be rationalized. We here present a model that integrates the current experimental knowledge and shows that under external forces, MTs can be converted into metastable circular states, explaining the recurrent observations of gliding rings.

Switchable tube model.-We will develop a simple yet generic elastic model of taxol stabilized MTs that principally revises their mechanical response. We make use of the following experimental facts that were established for taxol stabilized protofilaments and MTs, a protocol also used in the majority of the gliding experiments. Fact 1: The protofilament can coexist in at least two conformational states [36], one of them straight, the other one highly curved with a radius of curvature $20-30 \mathrm{~nm}$ [36-38]. Fact 2: The MT lattice displays two elongational states with one of them being about $2 \%$ shorter [39]. Both facts can be brought in connection by assuming that the curved conformation of a dimer is slightly shorter, cf. Fig. 2(a).

The MT is modeled, cf. Fig. 2(a), as a bilayered tubular structure with an inner and an outer layer of material (with (a)

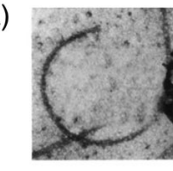

(c)

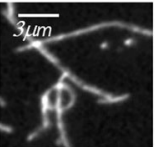

(b)

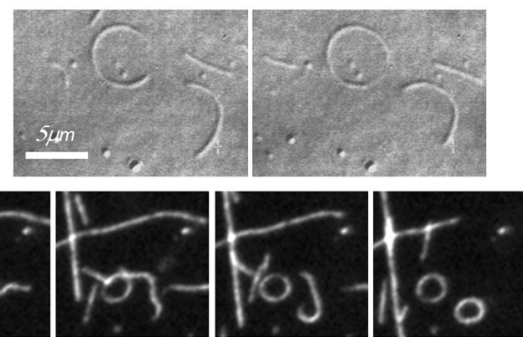

FIG. 1. (a)-(c) Mysterious ring formation of microtubules gliding on a kinesin motor carpet (from [18], [22], and [24]). 


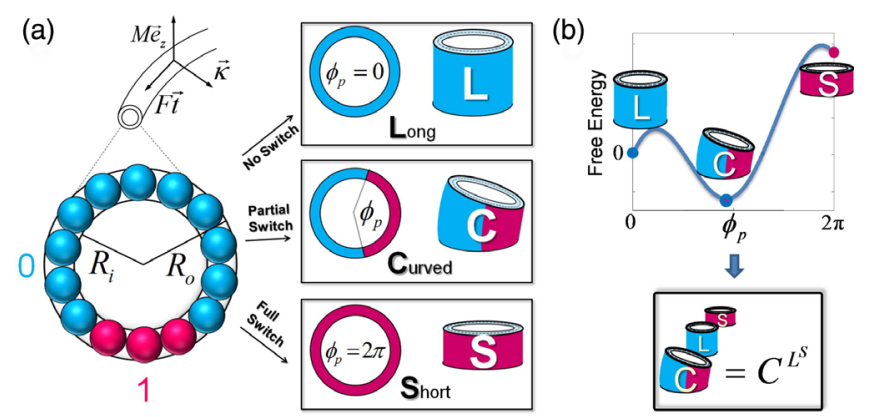

FIG. 2 (color online). (a) The bistable tube model with internal prestrains leading to three different lattice states: long and straight (" $L ")$, short and straight (" $S$ "), and curved ("C"). (b) A typical polymorphic energy landscape, cf. Eq. (3), with three metastable states and the associated polymorphic signature (with $C$ the ground state, and $L$ and $S$ "excited" states).

$R_{i, o}$ the inner and outer MT radii known from crystallography [1]). Both layers together represent the coarsegrained lattice of the tubulin dimers. The conformational "polymorphic" state of each tubulin [40] is described by a variable $\sigma$, assuming one of two states: either a straight one with $\sigma=0$ and vanishing preferred strain $\varepsilon_{\text {pref }}=0$. Or a curved state with $\sigma=1$ generating finite prestrain which, in general, has different values $\varepsilon_{\text {pref }}=\varepsilon_{i}$ or $\varepsilon_{o}$ in the two layers. These prestrains account for dimer curving and shortening. A tube section with a block of dimers switched to the curved ("1") state will endure prestress that can be partially relieved by global tube deformations, cf. Fig. 2(a): the MT will show axial length change and, remarkably, curving.

The MT cross section is parametrized in polar coordinates, $\vec{\rho}=\rho(\cos \phi, \sin \phi)$. Its energy density consists of two parts: first, the elastic energy $e_{\mathrm{el}}=$ $(Y / 2) \int_{R_{i}}^{R_{o}} d \rho \int_{0}^{2 \pi} \rho d \phi\left[\varepsilon(\rho, \phi)-\varepsilon_{\text {pref }}(\rho, \phi)\right]^{2}$, with $Y$ the Young's modulus. The strain is given by $\varepsilon(\rho, \phi)=-\vec{\kappa} \cdot \vec{\rho}+\bar{\varepsilon}$, the combination of pure stretching $\bar{\varepsilon}$ and (vectorial) curvature $\vec{\kappa}$. Second, each dimer can reduce its free energy by $|\Delta G|$ by switching to the 1 state. The respective energy of the cross section is $e_{\text {switch }}=$ $(\Delta G / b) \sum_{n=1}^{N} \sigma_{n}$ with $b \approx 8 \mathrm{~nm}$ the tubulin size and $N$ the number of protofilaments in the MT (typically 13). A negative free energy $\Delta G<0$ will favor the 1 state. Within the lattice, however, the switching of dimers will compete with the elastic energy, as well as external forces $F$ along the MT and torques $M$ acting on the cross section.

Since switched dimers effectively strongly attract each other within the lattice (as shown in [41]), we consider here the situation where all switched dimers form a single block. The angular size of the switched block, which we refer to as the "polymorphic variable" in the following, is denoted by $\phi_{p}$. It is this inner variable of the lattice that is often concealed from observation but can give rise to surprising effects. The total energy of a cross section with a block of $p$ curved dimers (defining $\phi_{p}=2 \pi(p / N)$ ), $e_{\mathrm{el}}+e_{\text {switch }}+M \kappa+F \bar{\epsilon}$, can then be calculated. In doing so a characteristic curvature is obtained,

$$
\kappa_{1}=\frac{8}{3 \pi} \frac{\varepsilon_{i}\left(R_{m}^{3}-R_{i}^{3}\right)+\varepsilon_{o}\left(R_{o}^{3}-R_{m}^{3}\right)}{R_{o}^{4}-R_{i}^{4}},
$$

where $R_{m}=\left(R_{i}+R_{o}\right) / 2$. Scaling the absolute value of curvature $\kappa=|\vec{\kappa}|$ by $\kappa_{1}$, energy by $B \kappa_{1}^{2}$, torque like $m=$ $M / B \kappa_{1}$ and tension like $\lambda=F / B \kappa_{1}^{2}$ we obtain [41]

$$
\tilde{e}_{\mathrm{tot}}=\frac{\kappa^{2}}{2}+\frac{a_{0} \bar{\varepsilon}^{2}}{2}-\kappa \sin \frac{\phi_{p}}{2}+a_{1} \phi_{p} \bar{\varepsilon}+m \kappa+\lambda \bar{\varepsilon}+\gamma \phi_{p} .
$$

The first two terms are of purely elastic origin, the next two are cross-coupling terms between elastic and polymorphic variables, and the last three are related to the action of generalized forces. $\gamma=a_{2}+\left(N \Delta G / 2 \pi b B \kappa_{1}^{2}\right)$ is the effective energy density of switching; it consists of an elastic penalty due to the lattice constraint, $a_{2}$ and of the free energy difference per length $\Delta G / b$ due to switching. $a_{0}, a_{1}$, and $a_{2}$ are completely determined dimensionless constants [41].

Using the experimental facts 1 and 2, we estimate the preferred strains to be $\varepsilon_{i}=0.7 \times 10^{-2}$ (tensile) and $\varepsilon_{o}=$ $-3.3 \times 10^{-2}$ (compressive), see [41] for details, consistent with an outwards protofilament curving and shortening. Using these values and Eq. (1), as an important cross-check we can estimate the characteristic curvature, which sets the scale for the gliding arcs and rings, to be $\kappa_{1} \simeq 1.1 \mu \mathrm{m}^{-1}$, consistent with the experimental observations [18,22-24].

To carve out the features of the energy, Eq. (2), we first consider imposed external force and torque. A minimization with respect to strain $\bar{\varepsilon}$ and curvature $\kappa$ (assuming these adjust to the external load) yields

$$
e_{\mathrm{tot}}\left(\phi_{p}\right)=-\frac{c_{p}}{2} \phi_{p}^{2}+f \phi_{p}-m \sin \frac{\phi_{p}}{2}-\frac{1}{2} \sin ^{2} \frac{\phi_{p}}{2},
$$

a function of torque $m$ and effective force $f=\gamma-c_{f} \lambda$. The dimensionless constants $c_{p} \simeq 0.1, c_{f} \simeq 2.5 \times 10^{-3}$ can be estimated for the given prestrains $\varepsilon_{i, o}$, and $\gamma$ to be between 0 and 1 for $|\Delta G|$ of the order of several kT (see [41] for details). The force $f$ contains the tension $\lambda$, but is also a function of lattice geometry and, importantly, of the switching energy $\Delta G$.

Equation (3) is the central result: it describes the energy of a MT cross section with partially switched tubulins and contains all the information needed to characterize the mechanical behavior of taxol-stabilized MTs under external loads. As a function of the acting force and torque, the "state diagram" of a MT cross section displays a number of distinct regions: the energy landscape can exhibit one, two, or three local minima, cf. Fig. 2(b). The possible states corresponding to these minima are given in Fig. 2(a): first, a 
state with no dimers switched $\left(\phi_{p}=0\right)$, which is long and straight (" $L ")$. Second, a state with all dimers switched $\left(\phi_{p}=2 \pi\right)$, which is shorter (hence we call it "S") and also straight. And finally a state having a block of dimers switched $\left(0<\phi_{p}<2 \pi\right.$, different $\phi_{p}$ are possible depending on $f, m$ ), and which is curved (" $C$ "). For further discussion we introduce an intuitive notation (polymorphic signature) capturing both the shape of the energy landscape and the actual state: possible are a single $X$, two $X^{Y}$, or three minima (states) $X^{Y^{Z}}$, with $X, Y, Z$ either one of $S, L, C$. Increasing energy values are indicated by ascending indices and the actual state by underlining the corresponding index. For instance, in $\underline{X}^{Y^{Z}}$ the ground state $X$ is populated, while in $X^{\underline{Y}}$ the metastable state $Y$.

The complete variety of polymorphic signatures physically allowed by $e_{\text {tot }}$ for given torque $m$ and effective force $f$ is summarized in the "polymorphic state diagram" in Fig. 3. Note that the simple model—considering two dimer states-leads to an extremely rich lattice behavior. There is an intrinsic symmetry with respect to the vertical line given by $f=\pi q c_{p}$ and passing through point $P$ : a mirror operation with respect to this line transforms the two straight states $S$ and $L$ into each other, leaving the curved state invariant. We will hence restrict the discussion to MTs initially in the $L$ state. The "polymorphic region," where stable or metastable curved states occur, lies between the two red curves (3) and (3)'. Outside of these curves, the behavior is indistinguishable from the one of a simple elastic beam or wormlike chain (WLC). Between the two blue curves, (2) and (2)', the curved state $C$ has lowest energy. Moreover, in the region above the point $P$ and between the

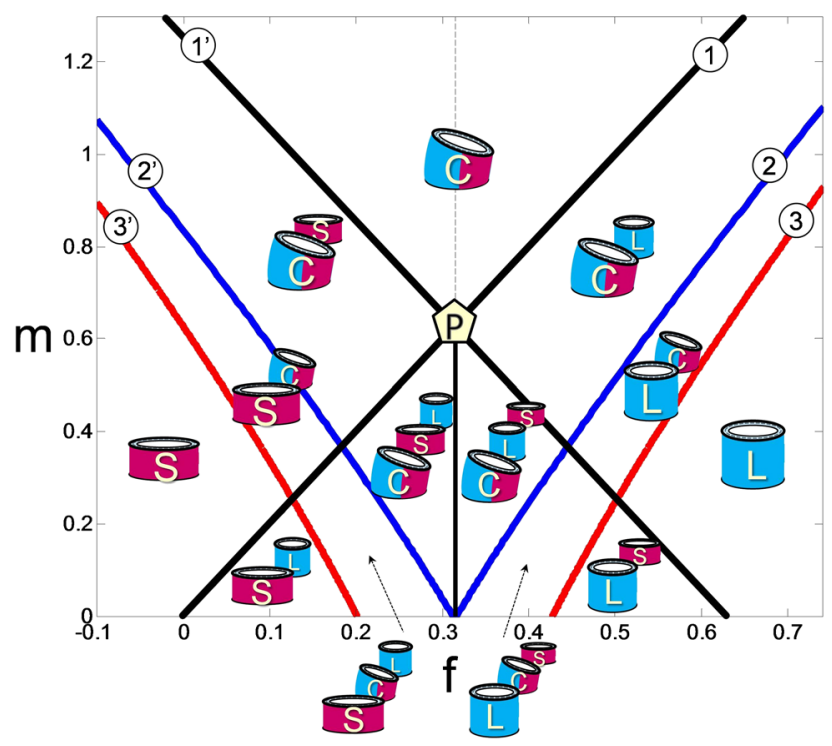

FIG. 3 (color online). The polymorphic state diagram for a MT cross section as a function of effective force $f$ and torque $m$, cf. Eq. (3). The diagram depends on the parameter $c_{p}$, estimated from the experimental measurements of protofilament curvature and MT lattice shortening (cf. main text). two black lines (1) (given by $m=2 f$ ) and (1)', state $C$ is even the only existing state. Finally, MTs are most sensitive to external loads close to point $P$, where state $C$ is the ground state in all surrounding regions. One expects "polymorphic behavior"-i.e., (parts of) MTs curved as in Fig. 1-to become visible when the respective cross sections are mechanically converted to the $C$ state. From the state diagram the necessary torque for this conversion can be estimated to be of the order of $10 \mathrm{pN} \mu \mathrm{m}$ (corresponding to $m=1$ in reduced units). As a single kinesin motor exerts forces of $2-5 \mathrm{pN}$ [1] on MTs with typical radius of curvature of $1 \mu \mathrm{m}$, this provides us with a first clue for understanding the MT's propensity to form rings in gliding assays, even for moderate motor coverage on the substrate.

Gliding-induced ring formation.-To investigate whether the found polymorphic signatures, emerging from the switchable internal structure of the MT, indeed explain ring formation, we generalized previous gliding assay modeling $[20,43]$ and study buckling events. Because of the confinement by the motors, the MT is described by a 2D space curve $\mathbf{r}(s)$, with $s$ the arclength. We assume that the MT's shape and the inner polymorphic variable $\phi_{p}(s)$ both follow relaxational dynamics

$$
\dot{\mathbf{r}}=\left(\frac{\mathbf{n n}}{\xi_{\perp}}+\frac{\mathbf{t t}}{\xi_{\|}}\right)\left[-\frac{\delta E}{\delta \mathbf{r}}+f_{m} \mathbf{t}\right], \quad \dot{\phi}_{p}=-\frac{1}{\zeta} \frac{\delta E}{\delta \phi_{p}},
$$

given by the polymorphic energy

$$
E=\int_{0}^{L} d s\left[B \kappa_{1}^{2} \tilde{e}_{\mathrm{tot}}+\frac{1}{2}\left(\mathbf{t}^{2}-1\right) \lambda+\frac{B_{p}}{2}\left(\partial_{s} \boldsymbol{\phi}_{p}\right)^{2}\right],
$$

where $\tilde{e}_{\text {tot }}(s)$ has been generalized to torques and forces, and consequently $\phi_{p}(s)$, depending on arclength. A length constraint [introducing the Lagrangian multiplier $\lambda(s)$, identified with the tension occurring in Eq. (2)] and a term penalizing variations in the polymorphic variable along the arclength have been added. The latter introduces a polymorphic stiffness $B_{p}$, modeling a certain cooperativity in lattice switching $[35,44]$. In the dynamic equations, we assumed anisotropic motor friction $\left(\xi_{\perp} \neq \xi_{\|}\right)$. $\zeta$ is associated to dissipation in the switching. $f_{m}$ is the local force density exerted by the kinesin motors transporting the filament along the local tangent $\mathbf{t}$. More details can be found in [41].

What happens if an initially straight MT gets stuck and buckles due to the action of motors? The answer depends on torque and force, varying in the course of buckling as follows [cf. blue curve in Fig. 4(d)]: initially, the MT is gliding freely, $f=\gamma$. Upon getting stuck, compressive force is rapidly created leading quasi-instantaneously to $f=\gamma-c_{f} \lambda$, followed by a slower buildup of torque due to the emerging curvature. As long as the MT tip remains stuck the torque increases while tension partially relaxes via buckling, see the part of the blue curve in Fig. 4(d) with 
(a)

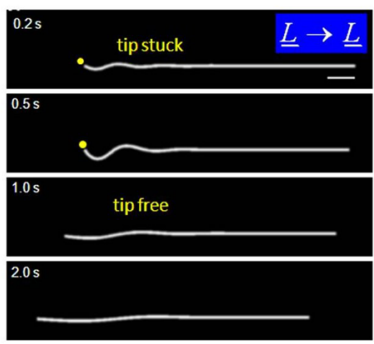

(c)

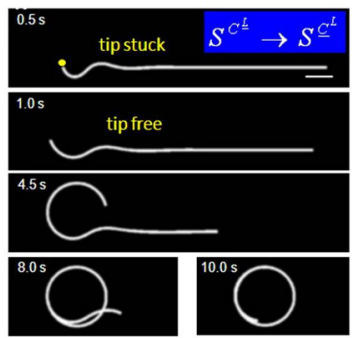

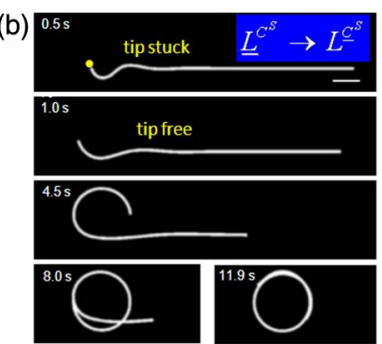

(e)

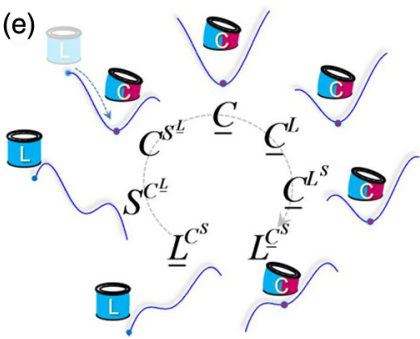

(d)

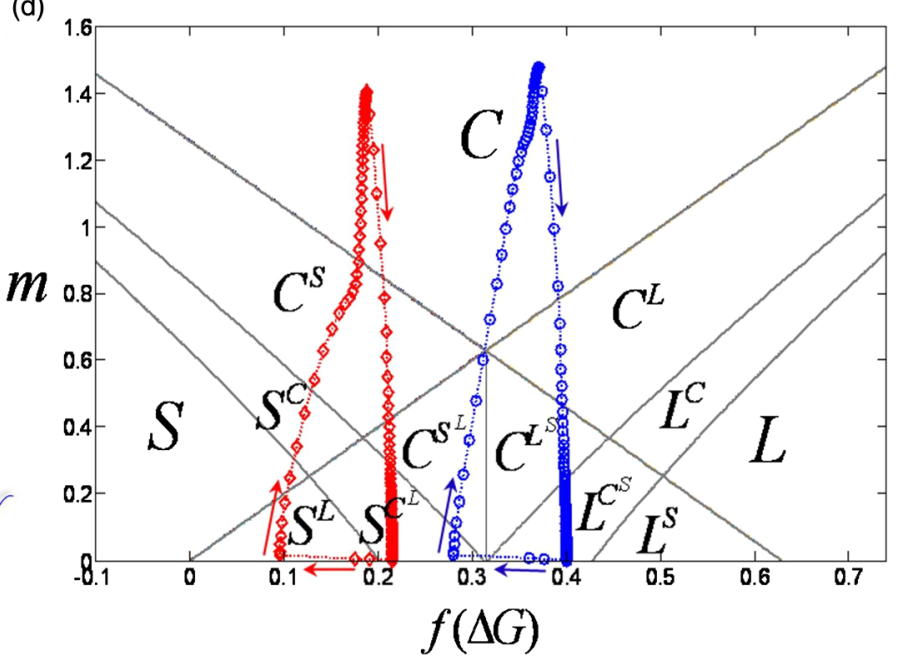

FIG. 4 (color online). Dynamic shape evolutions of gliding MTs that become temporarily stuck and buckle. All parameters are identical in (a)-(c), except for $\Delta G$ resulting in different effective forces $f$, cf. Fig. 3 and panel (d). (a) A MT far from the polymorphic region $(\Delta G=+1.7 \mathrm{kT}$ corresponding to $\gamma=1)$ behaves like a gliding WLC upon buckling and release. (b) A MT for which the buckling induces a switch to the curved state $(\Delta G=-5.2 \mathrm{kT}, \gamma=0.4)$. The finally formed ring is metastable; i.e., $L$ has lower energy than $C$. (c) A MT displaying multiple buckles that persist after unsticking and roll-up $(\Delta G=-7.3 \mathrm{kT}, \gamma=0.215)$. (d) Trajectories in the polymorphic state diagram corresponding to the dynamics shown in (b) (blue curve, circles) and (c) (red curve, diamonds), resulting in a hysteretic loop upon torque creation and release, cf. the sketch in (e). Scale bars in (a)-(c) are $1 \mu \mathrm{m}$, gliding velocity $v=1 \mu \mathrm{m} / \mathrm{s}$. All parameter values can be found in [41].

the upwards arrow. Upon tip release, the torque relaxes and one ends up at the starting point at $f=\gamma$ and $m \simeq 0$. This "loop" in the state diagram broadens and extends to higher torque values with increasing motor force $f_{m}$. Depending on force and torque induced during buckling, several distinct scenarios are possible:

For negligible dimer switching energies $\Delta G \simeq 0 \mathrm{kT}$ $(\gamma \simeq 0.85)$ or positive ones, a MT initially in the $L$ state buckles as a classical semiflexible filament (WLC) as shown in Fig. 4(a): meanders with force-dependent curvature emerge close to the tip and straighten again after unsticking. The same behavior is found for strongly negative values of $\Delta G \lesssim-15 \mathrm{kT}(\gamma \simeq-0.4)$, and a MT starting out from state $S$. In both cases, the induced torques are insufficient for crossing curve (1) or (1)', consequently the MT remains in the stable $L$ (or $S$ ) state.

For intermediate values of dimer switching energies $-6.3 \mathrm{kT} \lesssim \Delta G \lesssim-5.1 \mathrm{kT}(0.3 \lesssim \gamma \lesssim 0.4)$ the critical line (1) is crossed for high enough torques. Consequently, the $C$ state becomes populated at the filament tip, resulting in arclike buckles with curvature $\kappa \simeq \kappa_{1}$, see Fig. 4(b). Upon unsticking and concomitant torque decrease, state $L$ acquires lowest energy. Remarkably, despite $L$ being the ground state, the leading meander locks into the $C$ state, i.e., in the metastable configuration $L \underline{C}^{S}$. This scenario of a closed loop in the state diagram triggered by external forces and ending up in a metastable curved state, could be called mechanical hysteresis. The related path is shown as the blue curve in Fig. 4(d) and the concomitant changes in energy landscape and polymorphic signatures passed in
Fig. 4(e). Although state $C$ is only metastable, the whole MT eventually rolls up and converts to $C$ by simply following the motion of the tip in the course of gliding, cf. Fig. 4(b).

For dimer switching energies $-7.5 \mathrm{kT} \lesssim \Delta G \lesssim-6.3 \mathrm{kT}$ $(0.2 \lesssim \gamma \lesssim 0.3)$ the behavior is similar as just discussed, when the initial state $L$ is replaced by $S$. The MT converts into the $C$ state upon buckling, evolving from $\underline{S}^{C^{L}}$ into $S \underline{\underline{C}}^{C^{L}}$. Finally, Fig. 4(c) shows yet another scenario that gives rise to several stable arcs and takes place when $S$ is the ground state, yet the MT starts from the $L$ state [red curve in Fig. 4(d)].

The most sensitive parameter controlling polymorphic behavior is the free energy difference $\Delta G$ : changes in the range from 0 to $-10 \mathrm{kT}$ fundamentally alter the response to external forces. This suggests deep impact of various binding agents, like the Guanosine triphosphate-analogue Guanosine-5'-[( $\alpha \beta)$-methyleno] triphosphate [19], mitotic centromere-associated kinesin [36], or tau [45], either triggering or inhibiting polymorphic behavior. Our model yields estimated bounds for $\Delta G$ : if a MT forms metastable rings our analysis implies that $-7.5 \mathrm{kT} \lesssim \Delta G \lesssim-5 \mathrm{kT}$.

Conclusions. - The developed model principally revises the mechanics of MTs and explains their metastable curved states. It naturally comprises the classical semiflexible filament behavior at low loads. However, when the applied forces and torques exceed a threshold (tens of $\mathrm{pN}$ ), MTs convert to metastable curved conformations via a mechanical hysteresis loop. The polymorphic switching shares similarities with bacterial flagella [46-48]. 
The metastability-MTs "remember" force-induced curved shapes-puts them close to man-made shape memory materials [49]. The lattice switch renders MTs not only virtually unbreakable under typical cellular forces, but, moreover, provides them with a tunable response integrating mechanical and chemical stimuli. The major challenge will now be to unravel how the microtubule polymorphic switch is utilized in nature.

We thank A. Johner, K. J. Böhm, and D. Chrétien for stimulating discussions and A. Maloney, L. Herskowitz, and S. Koch for publishing and sharing their gliding assay image series open data. F. Z. thanks the German Science Foundation (DFG) for partial support via IRTG 1642 Soft Matter Science.

[1] J. Howard, Mechanics of Motor Proteins and the Cytoskeleton (Sinauer, Sunderland, 2001).

[2] L. A. Amos and W. G. Amos, Molecules of the Cytoskeleton (Guilford, New York, 1991).

[3] I. M. Kulić, A. E. X. Brown, H. Kim, C. Kural, B. Blehm, P. R. Selvin, P. C. Nelson, and V. I. Gelfand, Proc. Natl. Acad. Sci. USA 105, 10011 (2008).

[4] E. Karsenti and I. Vernos, Science 294, 543 (2001).

[5] C. P. Brangwynne, G. H. Koenderink, F. C. MacKintosh, and D. A. Weitz, Phys. Rev. Lett. 100, 118104 (2008).

[6] E. Meyrhöfer and J. Howard, Proc. Natl. Acad. Sci. U.S.A. 92, 574 (1995).

[7] C. Reuther, L. Hajdo, R. Tucker, A. A. Kasprzak, and S. Diez, Nano Lett. 6, 2177 (2006).

[8] L. Ionov, V. Bocharova, and S. Diez, Soft Matter 5, 67 (2009).

[9] T. Sanchez, D. Welch, D. Nicastro, and Z. Dogic, Science 333, 456 (2011).

[10] T. Sanchez, D. T. Chen, S. J. DeCamp, M. Heymann, and Z. Dogic, Nature (London) 491, 431 (2012).

[11] K. H. Downing and E. Nogales, Curr. Opin. Cell Biol. 10, 16 (1998).

[12] F. Gittes, B. Mickey, J. Nettleton, and J. Howard, J. Cell Biol. 120, 923 (1993).

[13] P. Venier, A. C. Maggs, M. F. Carlier, and D. Pantaloni, J. Biol. Chem. 269, 13353 (1994).

[14] H. Felgner, R. Frank, and M. Schliwa, J. Cell Sci. 109, 509 (1996).

[15] F. Pampaloni, G. Lattanzi, A. Jonas, T. Surrey, E. Frey, and E.-L. Florin, Proc. Natl. Acad. Sci. U.S.A. 103, 10248 (2006).

[16] K. M. Taute, F. Pampaloni, E. Frey, and E. L. Florin, Phys. Rev. Lett. 100, 028102 (2008).

[17] R. D. Vale, B. J. Schnapp, T. S. Reese, and M. P. Sheetz, Cell 40, 559 (1985).

[18] L. A. Amos and W. B. Amos, Journal of cell science Supplement 1991, 95 (1991).

[19] R. D. Vale, C. M. Coppin, F. Malik, F. J. Kull, and R. A. Milligan, J. Biol. Chem. 269, 23769 (1994).

[20] L. Bourdieu, T. Duke, M. Elowitz, D. Winkelmann, S. Leibler, and A. Libchaber, Phys. Rev. Lett. 75, 176 (1995).

[21] C. H. Aylett, J. Löwe, and L. A. Amos, Int. Rev. Cell Mol. Biol. 292, 1 (2011).
[22] K. J. Böhm, R. Stracke, W. Vater, and E. Unger, in Microand Nanostructures of Biological Systems, edited by H. J. Hein and G. Bischoff (Shaker Verlag, Aachen, 2001), pp. 153-165.

[23] P. Bieling, I. A. Telley, J. Piehler, and T. Surrey, EMBO Rep. 9, 1121 (2008)

[24] L. Liu, E. Tüzel, and J. L. Ross, J. Phys. Condens. Matter 23, 374104 (2011).

[25] R. Kawamura, A. Kakugo, K. Shikinaka, Y. Osada, and J. P. Gong, Biomacromolecules 9, 2277 (2008).

[26] H. Hess, J. Clemmens, C. Brunner, R. Doot, S. Luna, K.-H. Ernst, and V. Vogel, Nano Lett. 5, 629 (2005).

[27] I. Luria et al., Soft Matter 7, 3108 (2011).

[28] Y. Sumino, K. H. Nagai, Y. Shitaka, D. Tanaka, K. Yoshikawa, H. Chaté, and K. Oiwa, Nature (London) 483, 448 (2012).

[29] A. Maloney, L. J. Herskowitz, and S. J. Koch, PLoS One 6, e19522 (2011).

[30] T. J. Keating, J. G. Peloquin, V. I. Rodionov, D. Momcilovic, and G. G. Borisy, Proc. Natl. Acad. Sci. U.S.A. 94, 5078 (1997).

[31] A. D. Bicek, E. Tüzel, D. M. Kroll, and D. J. Odde, in Methods in Cell Biology, edited by Y. Wang and D. E. Discher (Elsevier, Amsterdam, y), pp. 237-268.

[32] C. P. Brangwynne et al., J. Cell Biol. 173, 733 (2006).

[33] C. Heussinger, M. Bathe, and E. Frey, Phys. Rev. Lett. 99, 048101 (2007).

[34] H. Mohrbach and I. M. Kulic, Phys. Rev. Lett. 99, 218102 (2007).

[35] H. Mohrbach, A. Johner, and I. M. Kulic, Phys. Rev. Lett. 105, 268102 (2010).

[36] C. Elie-Caille, F. Severin, J. Helenius, J. Howard, D. J. Muller, and A. A. Hyman, Curr. Biol. 17, 1765 (2007).

[37] T. Müller-Reichert, D. Chrétien, F. Severin, and A. A. Hyman, Proc. Natl. Acad. Sci. U.S.A. 95, 3661 (1998).

[38] Note that in [36] a third, less curved state with radius of curvature of $250 \mathrm{~nm}$ was also detected, which we neglect here as the MT shape will be dominated by the highly curved state.

[39] I. Arnal and R. H. Wade, Curr. Biol. 5, 900 (1995).

[40] Note that the MT lattice is assumed to have hydrolyzed the bulk of the GTP, i.e., we consider GDP-tubulin dimers, and it is stabilized by taxol.

[41] See Supplemental Material at http://link.aps.org/ supplemental/10.1103/PhysRevLett.114.148101, which includes Ref. [42], for derivations and more details on all equations, as well as for parameter estimates.

[42] S. Camalet and J. Jülicher, New J. Phys. 2, 24 (2000).

[43] F. Nedelec and D. Foethke, New J. Phys. 9, 427 (2007).

[44] H. Mohrbach, A. Johner, and I. M. Kulic, Eur. Biophys. J. 41, 217 (2012).

[45] A. Samsonov, J. Z. Yu, M. Rasenick, and S. V. Popov, J. Cell Sci. 117, 6129 (2004).

[46] N. C. Darnton and H. C. Berg, Biophys. J. 92, 2230 (2007).

[47] S. V. Srigiriraju and T. R. Powers, Phys. Rev. Lett. 94, 248101 (2005).

[48] H. Wada and R. R. Netz, Europhys. Lett. 82, 28001 (2008).

[49] K. Bhattacharya, Microstructure of Martensite: Why It Forms and How It Gives Rise to the Shape-Memory Effect (Oxford University Press, Oxford, 2003). 